\title{
VECTOR FORMULAE FOR NON-HOMOGENEOUS PRISMATIC BARS
}

\author{
ISTVÁN ECSEDI \\ Department of Mechanics, University of Miskolc \\ H-3515 Miskolc-Egyetemváros, Hungary \\ mechecs@uni-miskolc.hu \\ KornÉL DLuHi \\ Hewlett-Packard Hungary Ltd. \\ H-1117, Budapest, Alíz u. 1., Hungary \\ kornel.dluhi@hp.com
}

[Received: October 15, 2006]

\begin{abstract}
By using the Timoshenko type beam theory a simple solution is obtained for the bending-tension-shearing problem of non-homogeneous prismatic bars. Within this framework the elastic moduli can vary arbitrarily over the bar's cross section. Vector formulas for normal stress, shear flow and strain variables such as longitudinal strain and curvature for arbitrary cross section are derived. A vector-tensor formulation of the first-order shear deformation theory for thin-walled beams is presented. Applications of formulas obtained are illustrated in the case of thin-walled open and closed cross section deriving the formulas of shear centre and shear rigidity tensor.
\end{abstract}

Mathematical Subject Classification: 05C38, 15A15

Keywords: Bending, Non-homogeneous, Shear centre, Shear rigidity, Thin-walled, Timoshenko type beam

\section{INTRODUCTION}

This report is concerned with the tension (compression), bending and shearing of non-homogeneous prismatic bars. The analysis of the present report is based on the Timoshenko type beam theory in which the strain field is equivalent to assuming that plane transverse sections of prismatic bars remain plane after bending, shearing and tension (compression). The material of the bar is linearly elastic, non-homogeneous and isotropic. The material and geometrical properties do not depend on the axial coordinate $z$. The bar has non-homogeneity only in its cross section. It means that the Young's modulus $E$ and shear modulus $G$ may depend on the cross-sectional coordinates $x$ and $y$. This type of non-homogeneity is called cross-sectional nonhomogeneity. The definition of the cross-sectional non-homogeneity includes cases when the bar is a composite of different homogeneous materials, so that $E$ and $G$ are piecewise constants on the cross section. Types of these bars are the compound 
and reinforced bars. Their discontinuities in material properties should not affect the presented analysis.

A paper by Wang [1] presents the vectorial formulation of bending and shearing of homogeneous prismatic beams with arbitrary cross section. Wang [1 gives a new form of some known relationships changing the component (scalar) formulas by more compact vector formulas. For homogeneous and non-homogeneous beams the governing equations of pure bending are written in coordinate-free invariant forms by Stokes [2, 3]. In the paper by Stokes [4] a matrix formulation is developed to analyse the thermoelastic effect in pure bending of non-homogeneous prismatic bars. The formulation of Wang [1] and Stokes [2, 3] is based on the Bernoulli-Euler type beam theory, where the effect of the shear force to the deformations is neglected, it is assumed that the shear strains vanish.

The analysis of shear deformability of thin-walled beam is a coordinate free version, based on the shear factor tensor, is developed by Romano et al. [5]. Formulae of the position of shear centre derived in the present paper are different from them obtained by Romano et al. [5]. Our formulae are explicit expressions for the centre of shear, while paper by Romano et al. [5] gives implicit formulae for location of centre of shear in general case. Romano et al. [5] did not deal with the determination of the deflection curve of shear deformable thin-walled beams.

\section{Formulation of tension (COMPREssion) AND BENDing}

Consider a prismatic bar of arbitrary cross section. The longitudinal direction of the bar is denoted by the unit vector $\mathbf{e}_{z}$. The cross section of the bar is a connected planar domain $A$ orthogonal to vector $\mathbf{e}_{z}$. Under the action of axial force $N$ and the bending moment which is represented by vector $\mathbf{M}$ in the cross section the axial deformation of the bar according to the Timoshenko type beam theory can be written as

$$
\varepsilon_{z}=\varepsilon_{0}+\kappa \eta
$$

In equation 2.1), $\varepsilon_{z}$ is the normal strain in direction $z$ at point $P(x, y)$ and $\varepsilon_{0}$ is the value of $\varepsilon_{z}$ at the origin $O$ and $\kappa$ is the "rate of rotation with respect to $z$ " of the cross section about axis $\xi$. In the case of the Bernoulli-Euler type beam theory $\kappa$ is the curvature of the deformed longitudinal fibre for which $x=y=0$. The positions of axes $\xi$ and $\eta$ are unknown (Figure 1), they depend on the loading parameters which are the axial force and the bending moment vector.

Application of Hooke's law gives the formula of normal stress:

$$
\sigma_{z}=E(x, y) \varepsilon_{z}=E(x, y)\left[\varepsilon_{0}+\kappa \eta(x, y)\right] .
$$

Here, the function $E=E(x, y)$ may be continuous or discontinuous.

The relationship between the coordinates $x, y$ and $\xi, \eta$ is (Figure 1)

$$
\overrightarrow{O P}=\mathbf{R}=x \mathbf{e}_{x}+y \mathbf{e}_{y}=\xi \mathbf{n}+\eta \mathbf{m},
$$

where $\mathbf{n}, \mathbf{m}=\mathbf{e}_{z} \times \mathbf{n}$ are unit vectors, in which cross denotes the vectorial product according to Lurje [6] and Malvern [7]. 

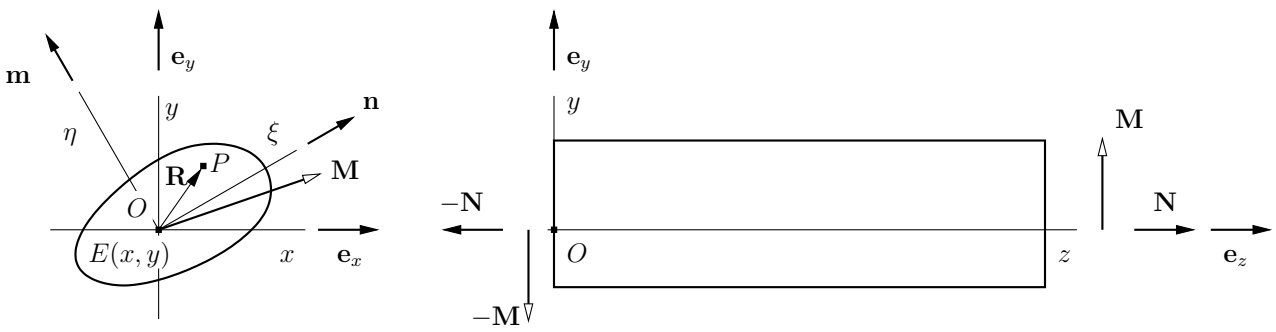

Figure 1. Non-homogeneous bar subjected to axial force and bending moment

The definition of the normal stress vector is as follows

$$
\mathbf{t}_{z}=\sigma_{z} \mathbf{e}_{z}=E \varepsilon_{0} \mathbf{e}_{z}+E \kappa \mathbf{n} \times \mathbf{R}
$$

since $\mathbf{n} \times \mathbf{R}=\eta \mathbf{e}_{z}$.

The axial force vector can be computed as

$$
\mathbf{N}=N \mathbf{e}_{z}=\int_{A} \mathbf{t}_{z} \mathrm{~d} A=\mathbf{e}_{z} \varepsilon_{0} \int_{A} E \mathrm{~d} A+\kappa \mathbf{n} \times \int_{A} E \mathbf{R} \mathrm{d} A .
$$

The following designations will be used

$$
S=\int_{A} E(x, y) \mathrm{d} A, \quad \mathbf{Q}=\int_{A} E(x, y) \mathbf{R} \mathrm{d} A .
$$

It is evident that

$$
N=\varepsilon_{0} S+\kappa \mathbf{n} \cdot\left(\mathbf{Q} \times \mathbf{e}_{z}\right),
$$

where the dot denotes the scalar product of two vectors [6, 7, 8,

The expression of the bending moment vector can be derived from the equation

$$
\mathbf{M}=\int_{A} \mathbf{R} \times \mathbf{t}_{z} \mathrm{~d} A .
$$

A simple calculation gives

$$
\mathbf{M}=\varepsilon_{0} \mathbf{Q} \times \mathbf{e}_{z}+\boldsymbol{J} \cdot \kappa \mathbf{n} .
$$

In equation 2.9 the $E$-weighted moment of inertia tensor of the cross section $A$ about point $O$ is introduced by the following definition

$$
\boldsymbol{J}=\int_{A} E(x, y)\left[\mathbf{1} R^{2}-\mathbf{R} \circ \mathbf{R}\right] \mathrm{d} A .
$$

In equation (2.10), the dyadic (tensorial) product of two vectors is denoted by a circle and $\mathbf{1}$ is the second order two-dimensional unit tensor [6, 7, 8. It is clear, that $\boldsymbol{J}$ is a positive definite symmetrical second order two-dimensional tensor. The validity of this statement follows from equation 2.10 .

The principal values of $\boldsymbol{J}$ are $J_{1} \geq J_{2}>0$ and the corresponding principal directions of $\boldsymbol{J}$ are given by unit vectors $\mathbf{e}_{1}$ and $\mathbf{e}_{2}$. We have

$$
\mathbf{e}_{1} \cdot \mathbf{e}_{2}=0 \quad \text { and } \quad \operatorname{det} \boldsymbol{J}=J_{1} J_{2} .
$$


The spectral decomposition of $\boldsymbol{J}$ and its inverse $\boldsymbol{J}^{-1}$ can be written as

$$
\boldsymbol{J}=J_{1} \mathbf{e}_{1} \circ \mathbf{e}_{1}+J_{2} \mathbf{e}_{2} \circ \mathbf{e}_{2}, \quad \boldsymbol{J}^{-1}=\frac{1}{J_{1}} \mathbf{e}_{1} \circ \mathbf{e}_{1}+\frac{1}{J_{2}} \mathbf{e}_{2} \circ \mathbf{e}_{2} .
$$

By the use of the equation written above an identity for $\boldsymbol{J}$ is derived which will be used later on. A simple computation gives

$$
\mathbf{e}_{z} \times \boldsymbol{J}^{-1} \times \mathbf{e}_{z}=-\left[\frac{1}{J_{1}} \mathbf{e}_{2} \circ \mathbf{e}_{2}+\frac{1}{J_{2}} \mathbf{e}_{1} \circ \mathbf{e}_{1}\right]
$$

where the following identities have been used for any dyad $\mathbf{a} \circ \mathbf{b}$ with arbitrary $\mathbf{c}$ and d vectors [6, 7, 8 ]

$$
\mathbf{c} \times(\mathbf{a} \circ \mathbf{b}) \times \mathbf{d}=(\mathbf{c} \times \mathbf{a}) \circ(\mathbf{b} \times \mathbf{d}) .
$$

The combination of equation 2.11) with equation 2.13 yields

$$
\mathbf{e}_{z} \times \boldsymbol{J}^{-1} \times \mathbf{e}_{z}=-\frac{\boldsymbol{J}}{\operatorname{det} \boldsymbol{J}}
$$

The aim is to get the relationship between the loading parameters $N, \mathbf{M}$ and strain variables $\varepsilon_{0}, \kappa \mathbf{n}$. Let the origin $O$ be the $E$-weighted centre $C$ of the cross section which is defined as (Figure 1)

$$
\int_{A} E(x, y) \mathbf{R} \mathrm{d} A=\mathbf{0}, \quad \mathbf{R}=\overrightarrow{C P} .
$$

In this case from equations 2.5 and 2.9 it follows that

$$
\varepsilon_{0}=\frac{N}{S}, \quad \kappa \mathbf{n}=\boldsymbol{J}^{-1} \cdot \mathbf{M}
$$

Combination of equation $(2.2)$ with equation 2.16 yields the formula of normal stress

$$
\sigma_{z}=E(x, y)\left[\frac{N}{S}+\mathbf{M} \cdot \boldsymbol{J}^{-1} \cdot\left(\mathbf{R} \times \mathbf{e}_{z}\right)\right] .
$$

Formula 2.17) in the case when $N=0$ can be considered as a generalisation of the classical bending (flexure) formula 9 .

\section{SheAr LOADING OF NON-HOMOGENEOUS BARS}

Figure 2 shows a bar segment which is loaded at its end cross sections so that $N=$ $0 \quad(0 \leq z \leq L)$. The cylindrical surface of the non-homogeneous bar is traction-free and there are no body forces. Equations of equilibrium of this bar element are as follows (Figure 2)

$$
\frac{\mathrm{d} \mathbf{F}}{\mathrm{d} z}=\mathbf{0}, \quad \frac{\mathrm{d} \mathbf{M}}{\mathrm{d} z}+\mathbf{e}_{z} \times \mathbf{F}=\mathbf{0} .
$$

Here, $\mathbf{F}$ is the internal force and $\mathbf{M}$ is the couple

$$
\mathbf{F}=\int_{A} \boldsymbol{\tau}_{z} \mathrm{~d} A, \quad \mathbf{M}=\left(\int_{A} \sigma \mathbf{R} \mathrm{d} A\right) \times \mathbf{e}_{z} .
$$




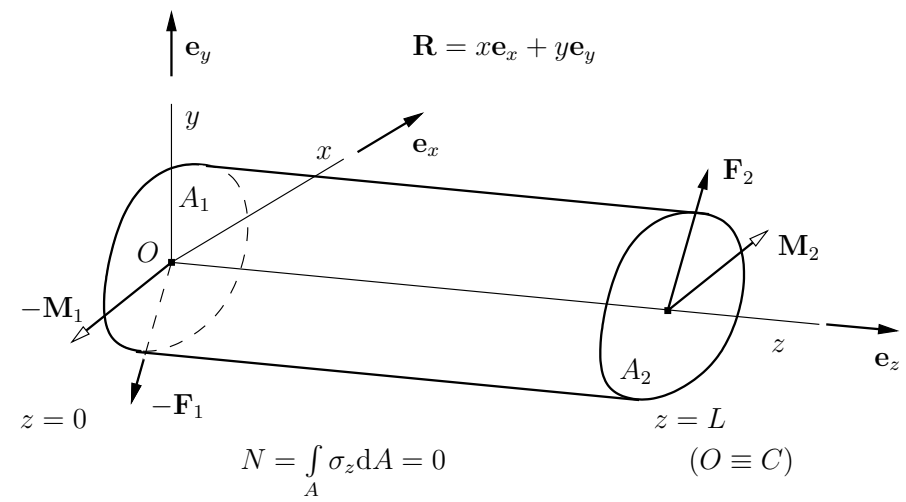

Figure 2. Bending and shear of a non-homogeneous bar

$\boldsymbol{\tau}_{z}=\tau_{x z} \mathbf{e}_{x}+\tau_{y z} \mathbf{e}_{y}$ is the shear stress vector, $\mathbf{e}_{x}$ and $\mathbf{e}_{y}$ are unit vectors in the direction of axes $x$ and $y$, respectively. It should be noticed that the applied loads at the end cross sections $A_{1}$ and $A_{2}$ satisfy the global conditions of equilibrium (Figure 2)

$$
\begin{gathered}
\mathbf{F}_{2}-\mathbf{F}_{1}=\mathbf{0}, \quad \mathbf{M}_{2}-\mathbf{M}_{1}+L \mathbf{e}_{z} \times \mathbf{F}_{2}=\mathbf{0}, \\
\int_{A_{2}} \mathbf{R} \times \boldsymbol{\tau}_{z}(x, y, L) \mathrm{d} A-\int_{A_{1}} \mathbf{R} \times \boldsymbol{\tau}_{z}(x, y, 0) \mathrm{d} A=\mathbf{0} .
\end{gathered}
$$

According to the equation of equilibrium the following can be written [6, 7 ]

$$
\begin{gathered}
\frac{\partial \tau_{x z}}{\partial x}+\frac{\partial \tau_{y z}}{\partial y}+\frac{\partial \sigma_{z}}{\partial z}=0 \quad(x, y) \in A \quad 0<z<L, \\
\tau_{x z} n_{x}+\tau_{y z} n_{y}=0 \quad(x, y) \in \partial A \quad 0<z<L .
\end{gathered}
$$

Here, $\partial A$ is the boundary curve of $A$ and $\mathbf{n}=n_{x} \mathbf{e}_{x}+n_{y} \mathbf{e}_{y}$ is the unit normal vector of boundary curve $\partial A$.

Consider the part $A^{*}$ of the cross section $A$ which is bounded by the line segment $\overline{H K}$ and the boundary segment $\partial A^{*}$ (Figure 3). From equation $\sqrt{3.5}$ it follows that

$$
\int_{A^{*}}\left(\frac{\partial \tau_{x z}}{\partial x}+\frac{\partial \tau_{y z}}{\partial y}\right) \mathrm{d} A=-\int_{A^{*}} \frac{\partial \sigma_{z}}{\partial z} \mathrm{~d} A .
$$

By using Stokes's theorem and the stress boundary condition it can be proved

$$
\begin{array}{r}
\int_{A^{*}}\left(\frac{\partial \tau_{x z}}{\partial x}+\frac{\partial \tau_{y z}}{\partial y}\right) \mathrm{d} A=\int_{\partial A^{*}}\left(\tau_{x z} n_{x}+\tau_{y z} n_{y}\right) \mathrm{d} s+\int_{\overline{H K}}\left(\tau_{x z} n_{x}+\tau_{y z} n_{y}\right) \mathrm{d} s \\
=\int_{\overline{H K}} \boldsymbol{\tau}_{z} \cdot \mathbf{m} \mathrm{d} s
\end{array}
$$

In equation (3.8 $s$ is an arc-length defined on $\partial A^{*} \cup \overline{H K}$ and $\mathbf{m}$ is the unit normal vector to line segment $\overline{H K}$ (Figure 3). The shear flow acting on the line segment $\overline{H K}$ 


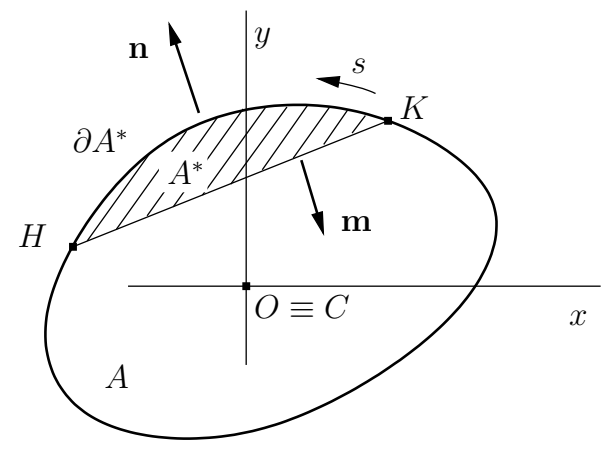

Figure 3. A sketch to the computation of shear flow

is denoted by $V_{H K}$ and it can be computed as

$$
V_{H K}=\int_{\overline{H K}} \boldsymbol{\tau}_{z} \cdot \mathbf{m} \mathrm{d} s=-\int_{A^{*}} \frac{\partial \sigma_{z}}{\partial z} \mathrm{~d} A .
$$

Note, that the unit of $V_{H K}$ is force/length. Assuming that $O \equiv C$ and the flexure formula is valid, from equation 2.17) with $N=0$ follows

$$
\frac{\partial \sigma_{z}}{\partial z}=E \frac{\mathrm{d} \mathbf{M}}{\mathrm{d} z} \cdot \boldsymbol{J}^{-1} \cdot\left(\mathbf{R} \times \mathbf{e}_{z}\right) .
$$

Substitution of equation 3.10 into equation 3.9 gives

$$
V_{H K}=-\left(\mathbf{F} \times \mathbf{e}_{z}\right) \cdot \boldsymbol{J}^{-1} \cdot\left(\mathbf{Q}_{H K} \times \mathbf{e}_{z}\right),
$$

where

$$
\mathbf{Q}_{H K}=\int_{A^{*}} E(x, y) \mathbf{R} \mathrm{d} A .
$$

Here, equation (3.1) has been used. The combination of equation 2.14 with equation (3.11) gives the final form of shear flow as

$$
V_{H K}=\mathbf{F} \cdot\left(\mathbf{e}_{z} \times \boldsymbol{J}^{-1} \times \mathbf{e}_{z}\right) \cdot \mathbf{Q}_{H K}=-\frac{\mathbf{F} \cdot \boldsymbol{J} \cdot \mathbf{Q}_{H K}}{\operatorname{det} \boldsymbol{J}} .
$$

\section{SheAR CENTRE AND SHEAR RIGIDITy OF A THIN-WALLED BEAM With OPEN CROSS SECTION}

The thin-walled beam of open cross section is loaded by a single force $\mathbf{F}=F_{x} \mathbf{e}_{x}+$ $F_{y} \mathbf{e}_{y}$ at the end cross section $A_{2}$, while the end cross section $A_{1}$ at $z=0$ is fixed (Figure 4). The centre line of the profile is denoted by $c$ and the thickness of the profile is indicated by $t=t(s)$, where $s$ is an arc-length coordinate defined on $c$. The origin of the cross-sectional coordinate system $O x y$ is the $E$-weighted centre of 


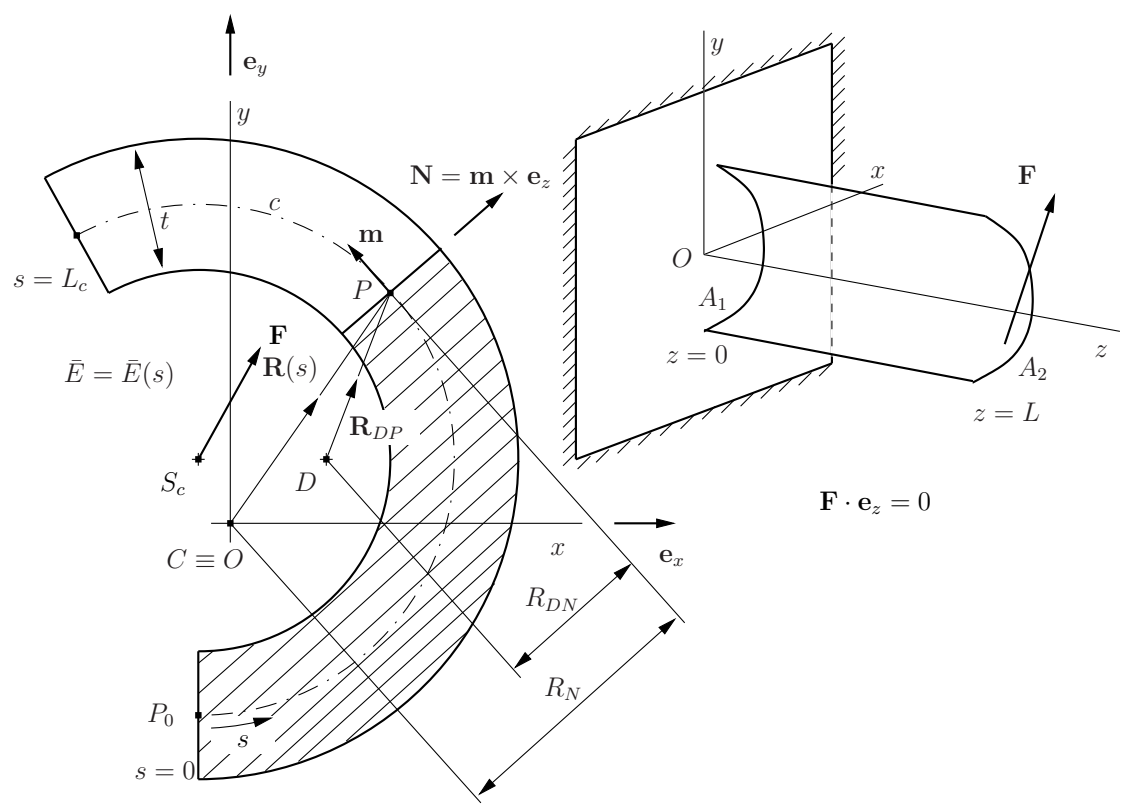

Figure 4. Thin-walled beam with open cross section

the cross section, that is $O \equiv C$. Let $\xi$ denote the thickness coordinate, and $\bar{E}$ is introduced by the definition

$$
\bar{E}(s)=\frac{1}{t(s)} \int_{-t / 2}^{t / 2} E \mathrm{~d} \xi
$$

The equation of the centre line of the open profile is

$$
\overrightarrow{O P}=\mathbf{R}(s)=X(s) \mathbf{e}_{x}+Y(s) \mathbf{e}_{y} .
$$

It is clear, that the $E$-weighted second moment of inertia tensor has the form

$$
\boldsymbol{J}=J_{x} \mathbf{e}_{x} \circ \mathbf{e}_{x}-J_{x y}\left(\mathbf{e}_{x} \circ \mathbf{e}_{y}+\mathbf{e}_{y} \circ \mathbf{e}_{x}\right)+J_{y} \mathbf{e}_{y} \circ \mathbf{e}_{y},
$$

where

$$
J_{x}=\int_{c} \bar{E} Y^{2} t \mathrm{~d} s, \quad J_{y}=\int_{c} \bar{E} X^{2} t \mathrm{~d} s, \quad J_{x y}=J_{y x}=\int_{c} \bar{E} X Y t \mathrm{~d} s .
$$

The $E$-weighted first moment of the shaded area about point $O$ is as follows (Figure 4 )

$$
\mathbf{Q}(s)=\int_{\widehat{P_{0} P}} \bar{E} \mathbf{R} t \mathrm{~d} s, \quad 0 \leq s \leq L_{c} .
$$

It follows that $\mathbf{Q}(0)=\mathbf{Q}\left(L_{c}\right)=\mathbf{0}$ since $O \equiv C$.

In equations 4.5 $L_{c}$ is the length of the middle curve $c$. 
An equivalent force-couple system at point $O$ to the shear flow

$$
V(s)=-\frac{\mathbf{F} \cdot \boldsymbol{J} \cdot \mathbf{Q}(s)}{\operatorname{det} \boldsymbol{J}}
$$

is

$$
\begin{gathered}
\mathbf{F}=\int_{c} V(s) \mathbf{m}(s) \mathrm{d} s \\
\mathbf{T}=\int_{c} \mathbf{R}(s) \times \mathbf{m}(s) V(s) \mathrm{d} s=\int_{c} V(s) \mathbf{R}(s) \times \mathrm{d} \mathbf{R},
\end{gathered}
$$

since

According to Figure 4

$$
\mathbf{m}(s)=\frac{\mathrm{d} \mathbf{R}}{\mathrm{d} s} .
$$

$$
\begin{aligned}
\mathbf{R} \times \mathrm{d} \mathbf{R}=\mathbf{R} \times \mathbf{m} \mathrm{d} s=\mathbf{R} \times\left(\mathbf{e}_{z}\right. & \times \mathbf{N}) \mathrm{d} s= \\
& =\mathbf{e}_{z}(\mathbf{R} \cdot \mathbf{N}) \mathrm{d} s-\mathbf{N}\left(\mathbf{R} \cdot \mathbf{e}_{z}\right) \mathrm{d} s=R_{N} \mathbf{e}_{z} \mathrm{~d} s
\end{aligned}
$$

where

$$
R_{N}=\mathbf{R} \cdot \mathbf{N} .
$$

Introducing equation 4.6 into equation 4.8 yields

$$
\mathbf{T}=\mathbf{e}_{z} \int_{c} V(s) R_{N} \mathrm{~d} s=-\frac{\mathbf{F} \cdot \boldsymbol{J} \cdot \int_{c} \mathbf{Q}(s) R_{N} \mathrm{~d} s}{\operatorname{det} \boldsymbol{J}} \mathbf{e}_{z} .
$$

Integration by parts gives the result

$$
\int_{c} \mathbf{Q}(s) R_{N}(s) \mathrm{d} s=[\mathbf{Q}(s) \omega(s)]_{0}^{L_{c}}-\int_{c} \frac{\mathrm{d} \mathbf{Q}}{\mathrm{d} s} \omega(s) \mathrm{d} s=-\int_{c} \bar{E} \mathbf{R} \omega \mathrm{d} s
$$

since

$$
\omega(s)=\int_{\widehat{P_{0} P}} R_{N} \mathrm{~d} s, \quad \frac{\mathrm{d} \mathbf{Q}}{\mathrm{d} s}=\bar{E}(s) \mathbf{R} t
$$

and $\mathbf{Q}(0)=\mathbf{Q}\left(L_{c}\right)=\mathbf{0}$. A new cross-sectional property $\mathbf{Q}_{\omega}$ is introduced as

$$
\mathbf{Q}_{\omega}=\int_{c} \bar{E}(s) \mathbf{R}(s) \omega(s) t(s) \mathrm{d} s .
$$

From equation 4.10 and 4.14 follows that

$$
\mathbf{T}=\frac{\mathbf{F} \cdot \boldsymbol{J} \cdot \mathbf{Q}_{\omega}}{\operatorname{det} \boldsymbol{J}} \mathbf{e}_{z}
$$

It is very easy to show that

$$
\mathbf{F} \times\left(\mathbf{e}_{z} \times\left(\boldsymbol{J} \cdot \mathbf{Q}_{\omega}\right)\right)=\mathbf{e}_{z}\left(\mathbf{F} \cdot \boldsymbol{J} \cdot \mathbf{Q}_{\omega}\right),
$$

since $\mathbf{F} \cdot \mathbf{e}_{z}=0$.

By the application of equation (4.17) a new form of the couple (torque) vector $\mathbf{T}$ can be derived

$$
\mathbf{T}=\mathbf{F} \times\left[\frac{\mathbf{e}_{z} \times \boldsymbol{J} \cdot \mathbf{Q}_{\omega}}{\operatorname{det} \boldsymbol{J}}\right] .
$$


The shear flow is caused by the shear force $\mathbf{F}$ whose point of application is the shear centre $S_{c}$. It is clear, that $\mathbf{T}$ can be computed as

$$
\mathbf{T}=\overrightarrow{O S_{c}} \times \mathbf{F} \text {. }
$$

Comparing two expressions of $\mathbf{T}$ which are given by equations 4.18 and 4.19 a vector formula can be obtained to determine the position of the shear centre

$$
\overrightarrow{O S_{c}}=-\frac{\mathbf{e}_{z} \times \boldsymbol{J} \cdot \mathbf{Q}_{\omega}}{\operatorname{det} \boldsymbol{J}}, \quad(O \equiv C) .
$$

For homogeneous beams, the scalar version of formula 4.20 is identical to which can be found in Vlasov's book [10].

The derivation of the shear stiffness of the open profile is based on the expression of shear strain energy. The shear strain energy per unit length of the non-homogeneous beam is 10, 11 .

$$
U_{s}=\frac{1}{2} \int_{c} \int_{-t / 2}^{t / 2} \frac{\tau_{z}^{2}}{G} \mathrm{~d} \xi \mathrm{d} s=\frac{1}{2} \int_{c} \frac{\tau^{2}}{\bar{G}} t \mathrm{~d} s=\frac{1}{2} \int_{c} \frac{[V(s)]^{2}}{\bar{G} t} \mathrm{~d} s .
$$

Here, $\bar{G}(s)$ is introduced by the next definition

$$
\frac{t(s)}{\bar{G}(s)}=\int_{-t / 2}^{t / 2} \frac{\mathrm{d} \xi}{G} .
$$

A simple computation shows that

$$
V^{2}=\left[\frac{\mathbf{F} \cdot \boldsymbol{J} \cdot \mathbf{Q}(s)}{\operatorname{det} \boldsymbol{J}}\right]^{2}=\frac{\mathbf{F} \cdot \boldsymbol{J} \cdot(\mathbf{Q} \circ \mathbf{Q}) \cdot \boldsymbol{J} \cdot \mathbf{F}}{(\operatorname{det} \boldsymbol{J})^{2}} .
$$

Defining the tensor $\boldsymbol{W}$ as

$$
\boldsymbol{W}=\int_{c} \frac{\mathbf{Q}(s) \circ \mathbf{Q}(s)}{\bar{G}(s) t(s)} \mathrm{d} s
$$

and using equation 4.23 it follows that

$$
U_{s}=\frac{1}{2} \mathbf{F} \cdot \boldsymbol{H}_{s} \cdot \mathbf{F}
$$

where $\boldsymbol{H}_{s}$ is the shear flexibility tensor

$$
\boldsymbol{H}_{s}=\frac{\boldsymbol{J} \cdot \boldsymbol{W} \cdot \boldsymbol{J}}{(\operatorname{det} \boldsymbol{J})^{2}} .
$$

The shear rigidity tensor $\boldsymbol{C}_{s}$ is the inverse of $\boldsymbol{H}_{s}$, that is

$$
\boldsymbol{C}_{s}=\boldsymbol{H}_{s}^{-1} \text {. }
$$

The principal directions of bending are the principal axes of $E$-weighted second moment of inertia tensor computed to point $C$. The principal directions of shear deformation are the principal axes of the shear rigidity (flexibility) tensor. From equation 4.26 it follows that in general, the principal directions of bending deformation and the principal directions of shear deformation have different orientations. The principal directions of bending and shear deformations coincide only if $\boldsymbol{J}$ and $\boldsymbol{W}$ have the same eigenvectors. In the case of symmetric cross sections this latter 

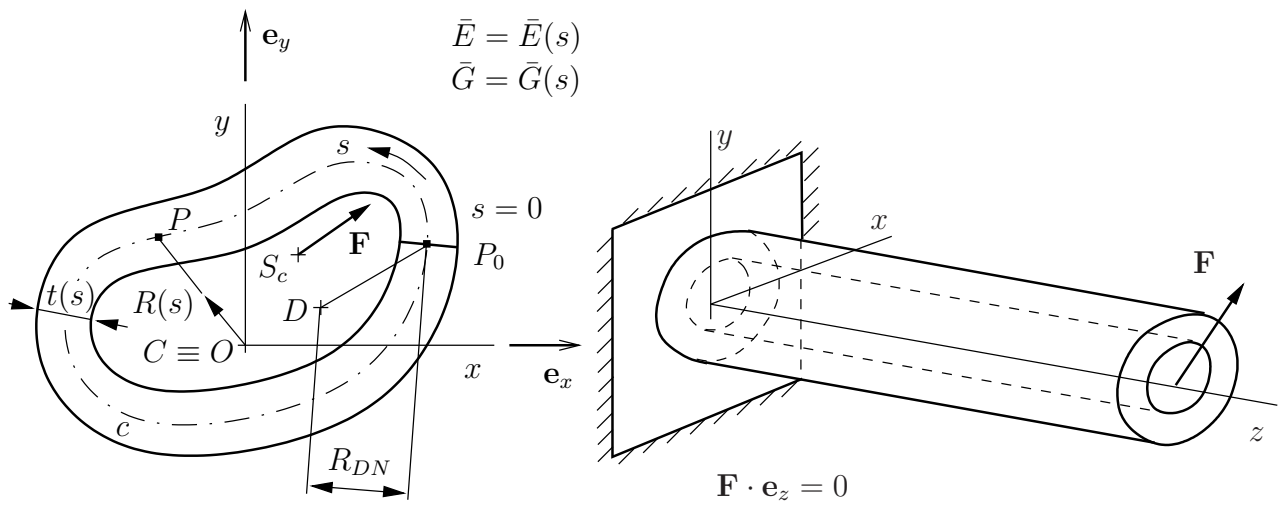

Figure 5. Thin-walled beam with closed cross section

statement is valid, that is the principal directions of bending and shear deformations coincide.

\section{Shear Centre and shear rigidity of a thin-Walled Beam With Closed CROSS SECTION}

For a single cell closed thin-walled tube shown in Figure 5 the shear flow $V=V(s)$ at any point $s$ is

$$
V(s)=V_{0}-\frac{\mathbf{F} \cdot \boldsymbol{J} \cdot \mathbf{Q}(s)}{\operatorname{det} \boldsymbol{J}},
$$

where $V_{0}$ is the value of $V$ at $s=0$ and $s$ is an arc-length coordinate measured along the centre line $c$. In order to find the shear flow caused by the shear force $\mathbf{F}$, it is necessary to determine $V_{0}$. In the literature of thin-walled beams it is customary to define the torsion-free bending of the tube as one in which the value of $V_{0}$ is chosen so, that the strain energy stored in the tube is minimal [11. This condition leads to the equation

$$
\int_{c} \frac{V(s)}{\bar{G} t} \mathrm{~d} s=0 .
$$

From equations (5.1) and 5.2 the following can be derived

$$
V_{0}=\frac{\mathbf{F} \cdot \boldsymbol{J} \cdot \int_{c} \frac{\mathbf{Q}(s)}{\bar{G} t} \mathrm{~d} s}{\operatorname{det} \boldsymbol{J} \int_{c} \overline{\mathrm{d} s}} \overline{\bar{G} t} .
$$

Note, that the thin-walled tube is axially homogeneous, i.e. all material and geometrical properties do not change in the direction of axis $z$.

The derivation of the next identity is based on integration by parts

$$
\int_{c} \frac{\mathbf{Q}(s)}{\bar{G} t} \mathrm{~d} s=-\int_{c}\left(\int_{0}^{s} \frac{\mathrm{d} \lambda}{\bar{G} t}\right) \bar{E} \mathbf{R} t \mathrm{~d} s .
$$


The point of application of the end load $\mathbf{F}$, at which the twist is not developed, is denoted by $S_{c}$. Point $S_{c}$ is the shear centre of thin-walled beams with closed profile. The torque about point $O \equiv C$ obtained from $\mathbf{F}$ is

$$
\mathbf{T}=\overrightarrow{O S_{c}} \times \mathbf{F} \text {. }
$$

The expression of the same torque in terms of shear flow in the case of torsion-free bending is as follows

$$
\mathbf{T}=\mathbf{e}_{z} \int_{c} R_{N}(s) V(s) \mathrm{d} s .
$$

From equations (5.1), 5.3), (5.4) and from the comparison of the results formulated in equations (5.5) and (5.6) follows

$$
\overrightarrow{O S_{c}}=-\frac{\mathbf{e}_{z} \times \boldsymbol{J} \cdot \mathbf{Q}_{\Omega}}{\operatorname{det} \boldsymbol{J}} \quad(O \equiv C),
$$

where

$$
\Omega(s)=\omega(s)-\frac{2 A_{c}}{\int_{c} \frac{\mathrm{d} s}{\bar{G} t}} \int_{0}^{s} \frac{\mathrm{d} \lambda}{\bar{G} t},
$$

$A_{c}$ is the area enclosed by the middle curve $c$,

$$
\mathbf{Q}_{\Omega}=\int_{c} \bar{E} \mathbf{R} \Omega t \mathrm{~d} s
$$

In order to get the expression of shear stiffness $\boldsymbol{c}_{s}$ the expression of shear strain energy per unit length of the non-homogeneous thin-walled tube is introduced, which is [10, 11 .

$$
U_{s}=\frac{1}{2} \int_{c} \frac{[V(s)]^{2}}{\bar{G} t} \mathrm{~d} s .
$$

Here,

$$
\begin{gathered}
{[V(s)]^{2}=\left[\frac{\mathbf{F} \cdot \boldsymbol{J} \cdot \mathbf{q}(s)}{\operatorname{det} \boldsymbol{J}}\right]^{2}=\frac{\mathbf{F} \cdot \boldsymbol{J} \cdot(\mathbf{q}(s) \circ \mathbf{q}(s)) \cdot \boldsymbol{J} \cdot \mathbf{F}}{(\operatorname{det} \boldsymbol{J})^{2}},} \\
\mathbf{q}(s)=\frac{\int_{c} \frac{\mathbf{Q}}{G t} \mathrm{~d} s}{\int_{c} \frac{\mathrm{d} s}{G t}}-\mathbf{Q}(s) .
\end{gathered}
$$

We introduce the tensor $\boldsymbol{w}$ as

$$
\boldsymbol{w}=\int_{c} \frac{\mathbf{q}(s) \circ \mathbf{q}(s)}{\bar{G}(s) t(s)} \mathrm{d} s .
$$

Inserting equations (5.11) and (5.13) into the formula of $U_{s}$ results in

$$
U_{s}=\frac{1}{2} \mathbf{F} \cdot \boldsymbol{h}_{s} \cdot \mathbf{F},
$$

where $\boldsymbol{h}_{s}$ is the shear flexibility tensor of the non-homogeneous thin-walled tube. Expression of $\boldsymbol{h}_{s}$ is

$$
\boldsymbol{h}_{s}=\frac{\boldsymbol{J} \cdot \boldsymbol{w} \cdot \boldsymbol{J}}{(\operatorname{det} \boldsymbol{J})^{2}} .
$$

The shear rigidity tensor $\boldsymbol{c}_{s}$ is the inverse of $\boldsymbol{h}_{s}$, that is

$$
\boldsymbol{c}_{s}=\boldsymbol{h}_{s}^{-1} \text {. }
$$


Following the discussion on the coincidence of principal directions of bending and shear deformations given at the end of Section 4 the next statement can be formulated. The principal directions of bending and shear deformations for the non-homogeneous thin-walled tube will be the same only if $\boldsymbol{J}$ and $\boldsymbol{w}$ have the same eigenvectors.

\section{Remark to the Computation of the Centre of Shear}

6.1. Open cross section. Here, a new formula is derived to obtain the position of the centre of shear $S_{c}$ relative to an arbitrary point $D$. According to Figure 4 and formula 4.12

$$
\overrightarrow{D S_{c}} \times \mathbf{F}=-\frac{\mathbf{F} \cdot \boldsymbol{J} \cdot \int_{c} \mathbf{Q}(s) R_{D N}(s) \mathrm{d} s}{\operatorname{det} \boldsymbol{J}} \mathbf{e}_{z},
$$

where $R_{D N}=\overrightarrow{D S_{c}} \cdot \mathbf{N}$. From equation 6.1 it follows that

$$
\mathbf{F} \cdot\left\{\mathbf{e}_{z} \times \overrightarrow{D S_{c}}+\frac{\boldsymbol{J} \cdot \int_{c} \mathbf{Q}(s) R_{D N}(s) \mathrm{d} s}{\operatorname{det} \boldsymbol{J}}\right\}=0 .
$$

Combination of equation $(6.2)$ with the next identity

$$
\left(\mathbf{e}_{z} \times \overrightarrow{D S_{c}}\right) \times \mathbf{e}_{z}=\overrightarrow{D S_{c}}
$$

gives

$$
\overrightarrow{D S_{c}}=\frac{\mathbf{e}_{z} \times \boldsymbol{J} \cdot \int_{c} \mathbf{Q}(s) R_{D N}(s) \mathrm{d} s}{\operatorname{det} \boldsymbol{J}} .
$$

6.2. Closed cross section. In the case of closed profile a new formula can be derived by the same method to determine the position of the centre of shear $S_{c}$ relative to an arbitrary point $D$. Here, equations (5.1) and (5.3) are used. A detailed computation yields the next result (Figure 5)

$$
\overrightarrow{D S_{c}}=\frac{\mathbf{e}_{z} \times \boldsymbol{J} \cdot \int_{c} \mathbf{Q}(s) R_{D N}(s) \mathrm{d} s}{\operatorname{det} \boldsymbol{J}}-\frac{\mathbf{e}_{z} \times \boldsymbol{J} \cdot \int_{c} \frac{\mathbf{Q}(s)}{G t} \mathrm{~d} s}{\operatorname{det} \boldsymbol{J}} \frac{2 A_{c}}{\int_{c} \frac{\mathrm{d} s}{\bar{G} t}} .
$$

Note, that in equations (6.4) and 6.5 and $\mathbf{Q}=\mathbf{Q}(s)$ refer to the $E$-weighted centre of the cross section.

\section{Deflection Curve of Shear Deformable thin-Walled NON-HOMOGENEOUS BEAMS}

It is assumed that the displacements and strains are small and the form of the displacement field is

$$
\mathbf{u}(x, y, z)=\mathbf{U}(z)+\left[w_{0}(z)+\left(\mathbf{e}_{z} \times \boldsymbol{\psi}(z)\right) \cdot \mathbf{R}\right] \mathbf{e}_{z},
$$

where $\mathbf{U}(z)=U(z) \mathbf{e}_{x}+V(z) \mathbf{e}_{y}$ is the transverse displacement vector of the $E$ weighted centre of the cross section and $w_{0}=w_{0}(z)$ is the axial displacement of the $E$-weighted centre of the cross section while $\boldsymbol{\psi}=\psi_{x}(z) \mathbf{e}_{x}+\psi_{y}(z) \mathbf{e}_{y}$ describes the 
"rigid" rotation of cross sections according to Timoshenko's beam theory. From the strain-displacement relationships of the linearized theory of elasticity [6, 7, 8, follows

$$
\begin{gathered}
\varepsilon_{x}=\varepsilon_{y}=\gamma_{x y}=0, \quad \varepsilon_{z}=\frac{\partial w_{0}}{\partial z}+\left(\mathbf{e}_{z} \times \frac{\mathrm{d} \boldsymbol{\psi}}{\mathrm{d} z}\right) \cdot \mathbf{R}, \\
\gamma_{x z}=\frac{\mathrm{d} U}{\mathrm{~d} z}+\left(\mathbf{e}_{z} \times \boldsymbol{\psi}\right) \cdot \mathbf{e}_{x}, \quad \gamma_{y z}=\frac{\mathrm{d} V}{\mathrm{~d} z}+\left(\mathbf{e}_{z} \times \boldsymbol{\psi}\right) \cdot \mathbf{e}_{y} .
\end{gathered}
$$

The shear strain vector $\gamma_{z}$ is introduced as

$$
\boldsymbol{\gamma}_{z}=\gamma_{x z} \mathbf{e}_{x}+\gamma_{y z} \mathbf{e}_{y}=\frac{\mathrm{d} \mathbf{U}}{\mathrm{d} z}+\mathbf{e}_{z} \times \boldsymbol{\psi}
$$

It follows from the obtained strain components that the displacement field formulated in equation (7.1) satisfies the requirements of Timoshenko's beam theory, i.e. the cross sections remain planes and the shear strains do not vanish. The shear strain vector on a cross section is constant, since it depends only on axial coordinate $z$. According to this statement the beam theory based on the displacement field $(7.1)$ is a first-order shear deformation beam theory [12. It should be emphasised that axis $z$ is the centre line of the non-homogeneous beam, that is it connects the $E$-weighted centre of the cross sections. Internal forces in a cross section can be decomposed into two parts as

$$
\mathbf{F}=\mathbf{S}+\mathbf{e}_{z} N, \quad \mathbf{S} \cdot \mathbf{e}_{z}=0
$$

where $N$ is the axial force and $\mathbf{S}$ is the shear force vector. According to equation 7.5 the applied distributed load is resolved as

$$
\mathbf{f}=\mathbf{s}+\mathbf{e}_{z} n, \quad \mathbf{s} \cdot \mathbf{e}_{z}=0 .
$$

Force equilibrium equations can be written in the form

$$
\frac{\mathrm{d} N}{\mathrm{~d} z}+n=0, \quad \frac{\mathrm{d} \mathbf{S}}{\mathrm{d} z}+\mathbf{s}=\mathbf{0} \quad 0<z<L .
$$

Next, it is assumed that $n(z)=0 \quad(0<z<L)$ and $N(0)=0$, that is $N(z)=0$ $(0 \leq z \leq L)$.

The moment of internal forces about point $S_{c}$ is $\mathbf{M}=\mathbf{M}(z)$. It is assumed that there is no torsional load present, that is

$$
\mathbf{M} \cdot \mathbf{e}_{z}=0 \quad \text { and } \quad \mathbf{m} \cdot \mathbf{e}_{z}=0 .
$$

In equation $7.8 \mathbf{m}=\mathbf{m}(z)$ is the moment of the applied distributed transverse load about point $S_{c}$. The moment equilibrium equation has the form

$$
\frac{\mathrm{d} \mathbf{M}}{\mathrm{d} z}+\mathbf{e}_{z} \times \mathbf{S}+\mathbf{m}=\mathbf{0} .
$$

From equations 2.1 and 7.2 it follows that

$$
\varepsilon_{z}=\varepsilon_{0}+\kappa \eta=\varepsilon_{0}+\left(\mathbf{e}_{z} \times \kappa \mathbf{n}\right) \cdot \mathbf{R}=\frac{\mathrm{d} w_{0}}{\mathrm{~d} z}+\left(\mathbf{e}_{z} \times \frac{\mathrm{d} \boldsymbol{\psi}}{\mathrm{d} z}\right) \cdot \mathbf{R},
$$

that is

$$
\varepsilon_{0}=\frac{\mathrm{d} w_{0}}{\mathrm{~d} z}, \quad \kappa \mathbf{n}=\frac{\mathrm{d} \boldsymbol{\psi}}{\mathrm{d} z}
$$


On the other hand, the following holds

$$
\varepsilon_{0}=\frac{N}{S}=0 \quad 0 \leq z \leq L
$$

in the present case. According to equations (7.11) and 7.12, without the loss of generality it can be assumed that

$$
w_{0}(z)=0 \quad 0 \leq z \leq L .
$$

Expression of $\mathbf{M}$ in terms of $\boldsymbol{\psi}$ is as follows

$$
\mathbf{M}=\boldsymbol{J} \cdot \frac{\mathrm{d} \boldsymbol{\psi}}{\mathrm{d} z} .
$$

In order to get the relationship between shear force $\mathbf{S}$ and shear strain $\gamma_{z}$ the expression of shear strain energy per unit length of non-homogeneous beams is considered

$$
U_{s}=\frac{1}{2} \int_{A} \boldsymbol{\tau}_{z} \cdot \gamma_{z} \mathrm{~d} A=\frac{1}{2} \mathbf{S} \cdot \boldsymbol{\gamma}_{z}=\frac{1}{2} \mathbf{S} \cdot \boldsymbol{H} \cdot \mathbf{S} .
$$

For open cross section $\boldsymbol{H}=\boldsymbol{H}_{s}$, and for closed cross section (tube) $\boldsymbol{H}=\boldsymbol{h}_{s}$. equation 7.15 yields

$$
\gamma_{z}=\boldsymbol{H} \cdot \mathbf{S} \quad \text { or } \quad \mathbf{S}=\boldsymbol{C} \cdot \boldsymbol{\gamma}_{z}
$$

where $\boldsymbol{C}=\boldsymbol{H}^{-1}$ is the shear rigidity tensor.

Here, we note, that the shear strain vector $\gamma_{z}$ represented by equation (7.4) is constant on the cross section, from this it follows that the shear stress vector $\boldsymbol{\tau}_{z}$ will also be constant. The discrepancy between the stress state compatible with the equilibrium equations (formula (3.13) ) and the constant stress state of the first order shear deformation theory can be overcome approximately by introducing the shear rigidity tensor to obtain the shear strain vector $\gamma_{z}$ according to equation (7.16).

From equation 7.9 it follows that

$$
\frac{\mathrm{d}}{\mathrm{d} z}\left(\mathbf{e}_{z} \times \mathbf{M}\right)-\mathbf{S}+\mathbf{e}_{z} \times \mathbf{m}=\mathbf{0} .
$$

Integration of equations $(7.7)$ and $(7.17)$ gives

$$
\begin{gathered}
\mathbf{S}=-\int_{0}^{z} \mathbf{s}\left(\xi_{1}\right) \mathrm{d} \xi_{1}+\mathbf{c}_{1}, \\
\mathbf{e}_{z} \times \mathbf{M}=-\int_{0}^{z} \int_{0}^{\xi_{2}} \mathbf{s}\left(\xi_{1}\right) \mathrm{d} \xi_{1} \mathrm{~d} \xi_{2}-\int_{0}^{z} \mathbf{e}_{z} \times \mathbf{m}\left(\xi_{1}\right) \mathrm{d} \xi_{1}+\mathbf{c}_{1} z+\mathbf{c}_{2} .
\end{gathered}
$$

Here, $\mathbf{c}_{1}$ and $\mathbf{c}_{2}$ are the constants of integration $\left(\mathbf{c}_{1} \cdot \mathbf{e}_{z}=\mathbf{c}_{2} \cdot \mathbf{e}_{z}=0\right)$. A new kinematical quantity $\phi=\phi(z)$ is introduced as

$$
\phi(z)=\mathbf{e}_{z} \times \boldsymbol{\psi}(z) .
$$

It is evident, that

$$
\psi(z)=-\mathbf{e}_{z} \times \phi(z) .
$$

A simple calculation shows that

$$
\mathbf{e}_{z} \times \mathbf{M}(z)=\mathbf{e}_{z} \times \boldsymbol{J} \cdot \frac{\mathrm{d} \boldsymbol{\psi}}{\mathrm{d} z}=-\left(\mathbf{e}_{z} \times \boldsymbol{J} \times \mathbf{e}_{z}\right) \cdot \frac{\mathrm{d} \boldsymbol{\phi}}{\mathrm{d} z}=(\operatorname{det} \boldsymbol{J}) \boldsymbol{J}^{-1} \cdot \frac{\mathrm{d} \boldsymbol{\phi}}{\mathrm{d} z} .
$$


Here, equation 2.14 has been used for " $\boldsymbol{J}^{-1}$ ". Inserting equation 7.22 into equation 7.19 yields

$$
\frac{\mathrm{d} \boldsymbol{\phi}}{\mathrm{d} z}=\frac{\boldsymbol{J}}{\operatorname{det} \boldsymbol{J}} \cdot\left\{-\int_{0}^{z} \int_{0}^{\xi_{2}} \mathbf{s}\left(\xi_{1}\right) \mathrm{d} \xi_{1} \mathrm{~d} \xi_{2}-\int_{0}^{z} \mathbf{e}_{z} \times \mathbf{m}\left(\xi_{1}\right) \mathrm{d} \xi_{1}+\mathbf{c}_{1} z+\mathbf{c}_{2}\right\} .
$$

Integrating equation 7.23 with respect to $z$ results in

$$
\begin{array}{r}
\phi(z)=\frac{\boldsymbol{J}}{\operatorname{det} \boldsymbol{J}} \cdot\left\{-\int_{0}^{z} \int_{0}^{\xi_{3}} \int_{0}^{\xi_{2}} \mathbf{s}\left(\xi_{1}\right) \mathrm{d} \xi_{1} \mathrm{~d} \xi_{2} \mathrm{~d} \xi_{3}-\int_{0}^{z} \int_{0}^{\xi_{2}} \mathbf{e}_{z} \times \mathbf{m}\left(\xi_{1}\right) \mathrm{d} \xi_{1} \mathrm{~d} \xi_{2}\right. \\
\left.+\frac{\mathbf{c}_{1}}{2} z^{2}+\mathbf{c}_{2} z\right\}+\mathbf{c}_{3} \cdot
\end{array}
$$

From equations (7.4), (7.16) and 7.18 the next equation can be derived

$$
\boldsymbol{H} \cdot \mathbf{S}=\boldsymbol{H} \cdot\left(-\int_{0}^{z} \mathbf{s}\left(\xi_{1}\right) \mathrm{d} \xi_{1}+\mathbf{c}_{1}\right)=\frac{\mathrm{d} \mathbf{U}}{\mathrm{d} z}+\boldsymbol{\phi} .
$$

Substituting $\phi=\phi(z)$ in equation 7.25 and integrating it once more with respect to $z$ results in

$$
\begin{array}{r}
\mathbf{U}(z)=-\frac{\boldsymbol{J}}{\operatorname{det} \boldsymbol{J}} \cdot\left\{-\int_{0}^{z} \int_{0}^{\xi_{4}} \int_{0}^{\xi_{3}} \int_{0}^{\xi_{2}} \mathbf{s}\left(\xi_{1}\right) \mathrm{d} \xi_{1} \mathrm{~d} \xi_{2} \mathrm{~d} \xi_{3} \mathrm{~d} \xi_{4}\right. \\
\left.-\int_{0}^{z} \int_{0}^{\xi_{3}} \int_{0}^{\xi_{2}} \mathbf{e}_{z} \times \mathbf{m}\left(\xi_{1}\right) \mathrm{d} \xi_{1} \mathrm{~d} \xi_{2} \mathrm{~d} \xi_{3}+\frac{1}{6} \mathbf{c}_{1} z^{3}+\frac{1}{2} \mathbf{c}_{2} z^{2}\right\}-\mathbf{c}_{3} z+\mathbf{c}_{4} \\
+\boldsymbol{H} \cdot\left(-\int_{0}^{z} \int_{0}^{\xi_{2}} \mathbf{s}\left(\xi_{1}\right) \mathrm{d} \xi_{1} \mathrm{~d} \xi_{2}+\mathbf{c}_{1} z\right)
\end{array}
$$

The transverse deflection vector $\mathbf{U}=\mathbf{U}(z)$ consists of two parts, $\mathbf{U}=\mathbf{U}_{B}+\mathbf{U}_{S}$, where the bending part $\mathbf{U}_{B}$ is

$$
\begin{aligned}
\mathbf{U}_{B} & =\frac{\boldsymbol{J}}{\operatorname{det} \boldsymbol{J}} \cdot\left\{\int_{0}^{z} \int_{0}^{\xi_{4}} \int_{0}^{\xi_{3}} \int_{0}^{\xi_{2}} \mathbf{s}_{0}\left(\xi_{1}\right) \mathrm{d} \xi_{1} \mathrm{~d} \xi_{2} \mathrm{~d} \xi_{3} \mathrm{~d} \xi_{4}\right. \\
& \left.+\int_{0}^{z} \int_{0}^{\xi_{3}} \int_{0}^{\xi_{2}} \mathbf{e}_{z} \times \mathbf{m}\left(\xi_{1}\right) \mathrm{d} \xi_{1} \mathrm{~d} \xi_{2} \mathrm{~d} \xi_{3}-\frac{1}{6} \mathbf{c}_{1} z^{3}-\frac{1}{2} \mathbf{c}_{2} z^{2}\right\}-\mathbf{c}_{3} z+\mathbf{c}_{4}
\end{aligned}
$$

and the shear part of $\mathbf{U}$ is $\mathbf{U}_{S}$

$$
\mathbf{U}_{S}=\boldsymbol{H} \cdot\left(-\int_{0}^{z} \int_{0}^{\xi_{2}} \mathbf{s}\left(\xi_{1}\right) \mathrm{d} \xi_{1} \mathrm{~d} \xi_{2}+\mathbf{c}_{1} z\right)
$$

The bending part $\mathbf{U}_{B}=\mathbf{U}_{B}(z)$ is the same that can be derived from the classical Euler-Bernoulli's beam theory. When the shear stiffness tends to infinity, the shear strain vector $\gamma_{z}$ goes to zero vector, that is

$$
\mathbf{e}_{z} \times \boldsymbol{\psi}=\boldsymbol{\phi}=-\frac{\mathrm{d} \mathbf{U}}{\mathrm{d} z} .
$$




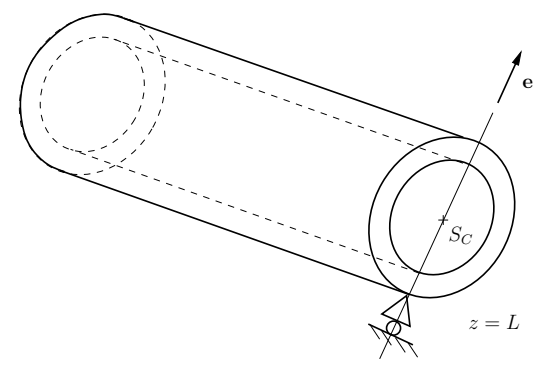

(a) Supported by one roller.

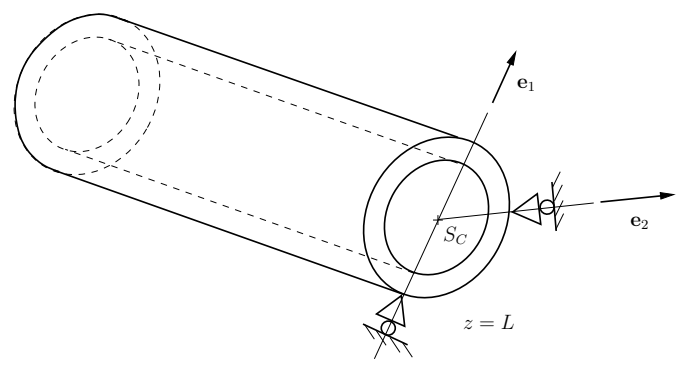

(b) Supported by two rollers.

Figure 6. Simply supported end cross sections $(a=L)$

If equation 7.29 is valid, the Timoshenko's beam theory reduces to the classical Euler-Bernoulli's beam theory [12].

Associated boundary conditions to equations (7.18), (7.19), 7.24) and (7.26) are as follows

- for fixed support: $\mathbf{U}(a)=\mathbf{0}, \quad \phi(a)=\mathbf{0} \quad(a=0$ or $a=L)$;

- for loaded end cross section: $\mathbf{M}(a)=\tilde{\mathbf{M}}, \quad \mathbf{S}(a)=\tilde{\mathbf{S}} \quad(a=0$ or $a=L)$, here $\tilde{\mathbf{M}}$ and $\tilde{\mathbf{S}}$ are prescribed;

- for free end cross section: $\mathbf{M}(a)=\mathbf{0}, \quad \mathbf{S}(a)=\mathbf{0} \quad(a=0$ or $a=L)$;

- for simply supported end cross section by one roller as shown in Figure 6(a). $\mathbf{U}(a) \cdot \mathbf{e}=0, \quad \mathbf{M}(a)=\mathbf{0}, \quad\left(\mathbf{e}_{z} \times \mathbf{S}(a)\right) \cdot \mathbf{e}=0 \quad(a=0$ or $a=L)$

- for end cross section supported by two rollers as shown in Figure $6(\mathrm{~b}) \mathbf{U}(a)=$ $\mathbf{0}, \quad \mathbf{M}(a)=\mathbf{0}$. The common point of lines of action of rollers is the centre of shear of considered end cross section.

\section{NUMERICAL EXAMPLES}

8.1. Shear centre and shear rigidity of a slitted rectangular tube. The thickness of cross section shown in Figure 7 is uniform but the walls are made of different materials. Using data given in Figure 7 results in

$$
\overrightarrow{D C}=\left(\begin{array}{c}
0.065 \\
0.098
\end{array}\right) \mathrm{m}, \boldsymbol{J}=\left(\begin{array}{cc}
721046 & -50597.6 \\
-50597.6 & 798352
\end{array}\right) \mathrm{Nm}^{2}, \overrightarrow{D S_{c}}=\left(\begin{array}{c}
0.219 \\
-0.053
\end{array}\right) \mathrm{m} .
$$

A simple computation gives

$$
\boldsymbol{H}_{s}=\left(\begin{array}{cc}
1.216 \mathrm{E}-07 & 7.633 \mathrm{E}-08 \\
7.633 \mathrm{E}-08 & 1.066 \mathrm{E}-07
\end{array}\right) \quad \frac{1}{\mathrm{~N}}, \quad \boldsymbol{C}_{s}=\left(\begin{array}{cc}
1.491 \mathrm{E}+07 & -1.067 \mathrm{E}+07 \\
-1.067 \mathrm{E}+07 & 1.702 \mathrm{E}+07
\end{array}\right) \mathrm{N} .
$$

The principal directions of bending deformation (eigenvectors of $\boldsymbol{J}$ ) are

$$
\mathbf{b}_{1}=\left(\begin{array}{c}
-0.896398 \\
-0.44325
\end{array}\right), \quad \mathbf{b}_{2}=\left(\begin{array}{c}
-0.44325 \\
0.896398
\end{array}\right)
$$




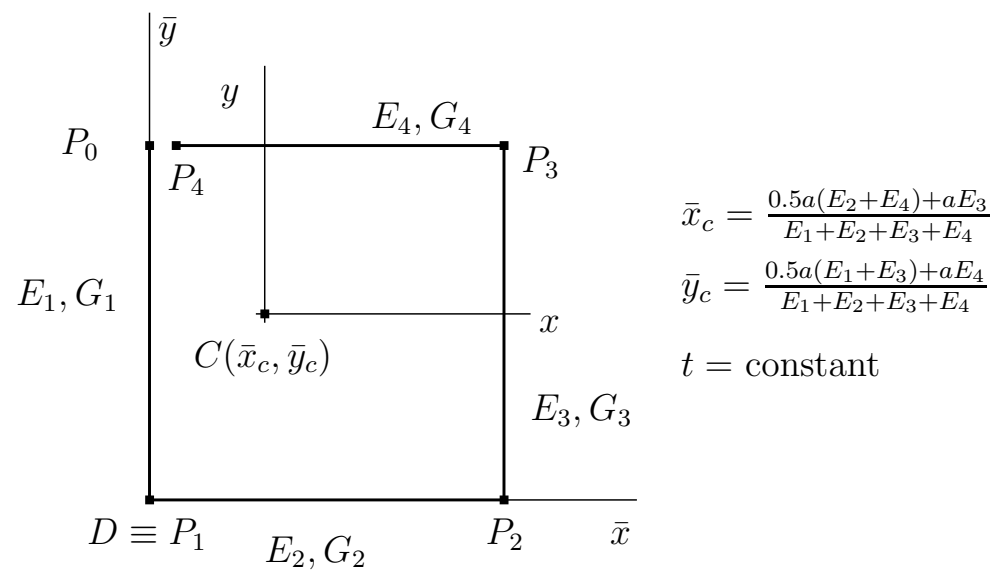

Figure 7. Slitted rectangular thin-walled tube. Data: $a=160 \mathrm{~mm}$, $t=2 \mathrm{~mm}, E_{1}=E_{4}=2.1 \times 10^{5} \mathrm{MPa}, E_{2}=0.7 \times 10^{5} \mathrm{MPa}, E_{3}=$ $1.05 \times 10^{5} \mathrm{MPa}, G_{1}=G_{4}=0.8 \times 10^{5} \mathrm{MPa}, G_{2}=0.27 \times 10^{5} \mathrm{MPa}$, $G_{3}=0.39 \times 10^{5} \mathrm{MPa}, \overline{P_{0} P_{1}}=\overline{P_{1} P_{2}}=a$

The principal directions of shear deformation (eigenvectors of $\boldsymbol{C}_{s}$ ) are

$$
\mathbf{S}_{1}=\left(\begin{array}{c}
-0.741031 \\
-0.67147
\end{array}\right), \quad \mathbf{S}_{2}=\left(\begin{array}{c}
0.67147 \\
-0.741031
\end{array}\right)
$$

In this example the principal directions of bending and shear deformations are significantly different.

8.2. Shear centre and shear rigidity of a closed rectangular tube. The middle curve of the closed tube is the same as above (Figure 7), but $P_{0} \equiv P_{4}$, i.e. there is no cut at point $P_{0}$. By the use of data given in Figure 7 the next results are obtained

$$
\begin{gathered}
\overrightarrow{D S_{c}}=\left(\begin{array}{c}
0.054 \\
0.123
\end{array}\right) \mathrm{m}, \quad \boldsymbol{h}_{s}=\left(\begin{array}{cc}
3.775 \mathrm{E}-08 & -1.841 \mathrm{E}-09 \\
-1.841 \mathrm{E}-09 & 3.381 \mathrm{E}-08
\end{array}\right) \frac{1}{\mathrm{~N}}, \\
\boldsymbol{c}_{s}=\left(\begin{array}{ll}
2.655 \mathrm{E}+07 & 1.446 \mathrm{E}+06 \\
1.446 \mathrm{E}+06 & 2.965 \mathrm{E}+07
\end{array}\right) \mathrm{N} .
\end{gathered}
$$

The principal directions of bending deformation for open and closed cross sections are the same. The principal directions of shear deformation (eigenvectors of $\boldsymbol{c}_{s}$ ) are

$$
\mathbf{s}_{1}=\left(\begin{array}{c}
-0.930169 \\
0.367131
\end{array}\right), \quad \mathbf{s}_{2}=\left(\begin{array}{c}
-0.367131 \\
-0.930169
\end{array}\right)
$$

8.3. Deflection curve of a cantilever thin-walled beam with open cross section. Consider a cantilever beam of length $L$ fixed at $z=0$ (Figure 8). The data of the "L" shape cross section is obtained from the data of the cross section shown in Figure 7. Here, $a=160 \mathrm{~mm}, t=2 \mathrm{~mm}, E_{1}=2.1 \times 10^{5} \mathrm{MPa}, E_{2}=0.7 \times 10^{5} \mathrm{MPa}$, 

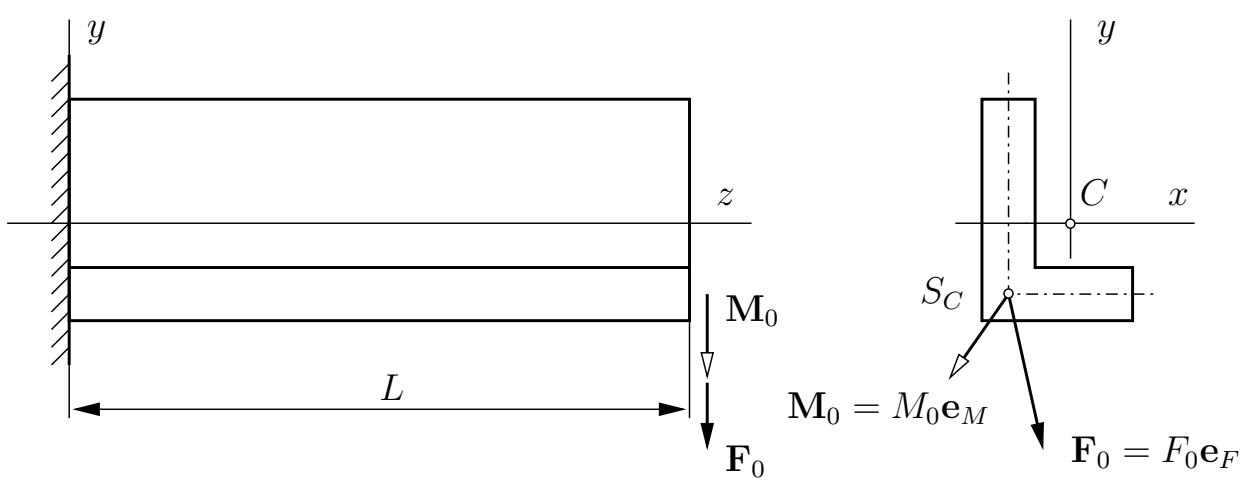

Figure 8. Cantilever thin-walled beam with open profile

$E_{3}=E_{4}=0, G_{1}=0.8 \times 10^{5} \mathrm{MPa}, G_{2}=0.27 \times 10^{5} \mathrm{MPa}, G_{3}=G_{4}=0$. The determination of the deflection vector $\mathbf{U}=\mathbf{U}(z)$ and the vector $\phi=\phi(z)$ is based on equations 7.18, 7.19, 7.24, 7.27) and the associated boundary conditions

$$
\mathbf{U}(0)=\mathbf{0}, \quad \phi(0)=\mathbf{0}, \quad \mathbf{S}(L)=\mathbf{F}_{0}, \quad \mathbf{M}(L)=\mathbf{M}_{0} .
$$

It is clear, that in the present problem $\mathbf{s}=\mathbf{0}$ and $\mathbf{m}=\mathbf{0}$. A detailed computation gives the next results:

$$
\begin{gathered}
\mathbf{c}_{1}=F_{0} \mathbf{e}_{F}, \quad \mathbf{c}_{2}=\mathbf{e}_{z} \times \mathbf{e}_{M} M_{0}-L F_{0} \mathbf{e}_{F}, \quad \mathbf{c}_{3}=\mathbf{0}, \quad \mathbf{c}_{4}=\mathbf{0} ; \\
\boldsymbol{\phi}(z)=\frac{\boldsymbol{J}}{\operatorname{det} \boldsymbol{J}} \cdot\left\{F_{0}\left(\frac{z^{2}}{2}-L z\right) \mathbf{e}_{F}+M_{0} z \mathbf{e}_{z} \times \mathbf{e}_{M}\right\}, \\
\mathbf{U}(z)=-\frac{\boldsymbol{J}}{\operatorname{det} \boldsymbol{J}} \cdot\left\{F_{0}\left(\frac{z^{3}}{6}-\frac{L z^{2}}{2}\right) \mathbf{e}_{F}+\frac{M_{0} z^{2}}{2} \mathbf{e}_{z} \times \mathbf{e}_{M}\right\}+z F_{0} \boldsymbol{H} \cdot \mathbf{e}_{F} .
\end{gathered}
$$

Table 1 illustrates the effect of shear to the end displacement of the cantilever thin-walled non-homogeneous beam with open profile.

8.4. Deflection curve of a fixed-fixed thin-walled beam with open cross section. Figure 9 shows the fixed-fixed supported thin-walled beam and its load. The applied load is acting on the whole length of the thin-walled beam. The intensity of the applied load is

$$
\mathbf{s}(z)=\mathbf{f}_{0}=f_{0} \mathbf{e}_{f}, \quad\left(f_{0}, \mathbf{e}_{f} \text { are constants }\right) .
$$

For a fixed-fixed end beam the boundary conditions are as follows

$$
\mathbf{U}(0)=\mathbf{0}, \quad \phi(0)=\mathbf{0}, \quad \mathbf{U}(L)=\mathbf{0}, \quad \phi(L)=\mathbf{0} .
$$

Application of equations (7.24), 7.27) and the first two of the boundary conditions mentioned above gives

$$
\phi(z)=\frac{\boldsymbol{J}}{\operatorname{det} \boldsymbol{J}} \cdot\left\{-\frac{f_{0} z^{3}}{6} \mathbf{e}_{f}+\frac{\mathbf{c}_{1}}{2} z^{2}+\mathbf{c}_{2} z\right\},
$$


Table 1. End deflection of the cantilever beam caused by a single force $\left(M_{0}=0, \mathbf{e}_{F}=\cos \alpha \mathbf{e}_{x}+\sin \alpha \mathbf{e}_{y}, L=2 \mathrm{~m}\right)$

\begin{tabular}{cccc}
\hline$\alpha$ & $\mathrm{U}(L) / F_{0}\left[\frac{\mathrm{m}}{\mathrm{N}}\right]$ & $\mathrm{U}_{B}(L) / F_{0}\left[\frac{\mathrm{m}}{\mathrm{N}}\right]$ & $\mathbf{U}_{S}(L) / F_{0}\left[\frac{\mathrm{m}}{\mathrm{N}}\right]$ \\
\hline 0 & $\left(\begin{array}{c}2.47073 \mathrm{E}-05 \\
1.0477 \mathrm{E}-05\end{array}\right)$ & $\left(\begin{array}{l}2.44088 \mathrm{E}-05 \\
1.04606 \mathrm{E}-05\end{array}\right)$ & $\left(\begin{array}{c}2.98525 \mathrm{E}-07 \\
1.6429 \mathrm{E}-08\end{array}\right)$ \\
\hline$\frac{\pi}{6}$ & $\left(\begin{array}{l}2.66356 \mathrm{E}-05 \\
1.66851 \mathrm{E}-05\end{array}\right)$ & $\left(\begin{array}{l}2.63689 \mathrm{E}-05 \\
1.66151 \mathrm{E}-05\end{array}\right)$ & $\left(\begin{array}{l}2.66745 \mathrm{E}-07 \\
7.00265 \mathrm{E}-08\end{array}\right)$ \\
\hline$\frac{\pi}{3}$ & $\left(\begin{array}{c}2.1427 \mathrm{E}-05 \\
1.84224 \mathrm{E}-05\end{array}\right)$ & $\left(\begin{array}{l}2.12635 \mathrm{E}-05 \\
1.83176 \mathrm{E}-05\end{array}\right)$ & $\left(\begin{array}{c}1.6349 \mathrm{E}-07 \\
1.04861 \mathrm{E}-07\end{array}\right)$ \\
\hline$\frac{\pi}{2}$ & $\left(\begin{array}{c}1.0477 \mathrm{E}-05 \\
1.52235 \mathrm{E}-05\end{array}\right)$ & $\left(\begin{array}{l}1.04606 \mathrm{E}-05 \\
1.51119 \mathrm{E}-05\end{array}\right)$ & $\left(\begin{array}{c}1.6429 \mathrm{E}-08 \\
1.11597 \mathrm{E}-07\end{array}\right)$ \\
\hline$\frac{5 \pi}{6}$ & $\left(\begin{array}{c}-1.61586 \mathrm{E}-05 \\
-1.4616 \mathrm{E}-06\end{array}\right)$ & $\left(\begin{array}{l}-1.59083 \mathrm{E}-05 \\
-1.50318 \mathrm{E}-06\end{array}\right)$ & $\left(\begin{array}{c}-2.50316 \mathrm{E}-07 \\
4.15707 \mathrm{E}-08\end{array}\right)$ \\
\hline$\frac{2 \pi}{3}$ & $\left(\begin{array}{c}-3.28028 \mathrm{E}-06 \\
7.94544 \mathrm{E}-06\end{array}\right)$ & $\left(\begin{array}{c}-3.14525 \mathrm{E}-06 \\
7.85701 \mathrm{E}-06\end{array}\right)$ & $\left(\begin{array}{c}-1.35035 \mathrm{E}-07 \\
8.84316 \mathrm{E}-08\end{array}\right)$ \\
\hline
\end{tabular}
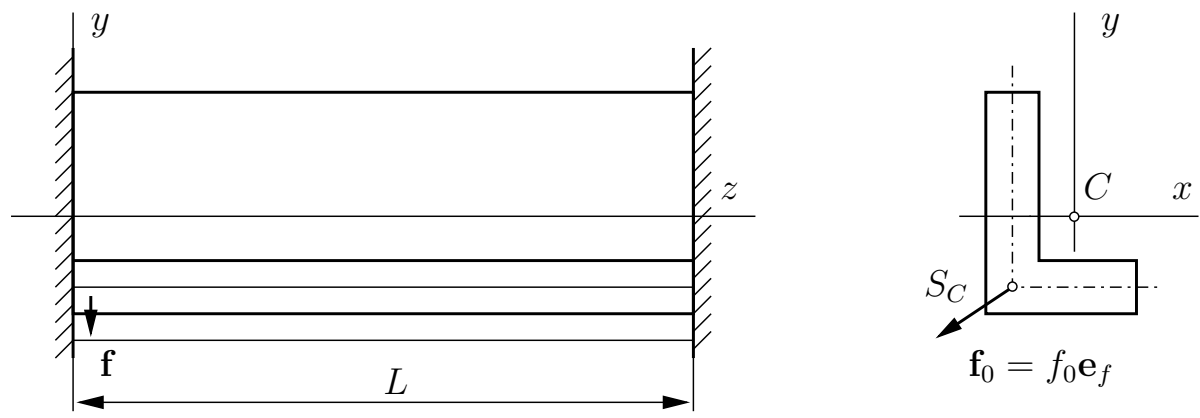

Figure 9. Uniform load on a fixed-fixed supported thin-walled beam with open profile

$$
\mathbf{U}(z)=\frac{\boldsymbol{J}}{\operatorname{det} \boldsymbol{J}} \cdot\left\{\frac{f_{0} z^{4}}{24} \mathbf{e}_{f}-\frac{\mathbf{c}_{1}}{6} z^{3}-\frac{\mathbf{c}_{2}}{2} z^{2}\right\}+\boldsymbol{H} \cdot\left\{-\frac{f_{0} z^{2}}{2} \mathbf{e}_{f}+\mathbf{c}_{1} z\right\} .
$$

In the present problem $\mathbf{c}_{3}=\mathbf{c}_{4}=\mathbf{0}$. From the boundary conditions $\mathbf{U}(L)=$ $\phi(L)=\mathbf{0}$ it can be deduced

$$
\mathbf{c}_{1}=\frac{f_{0} L}{2} \mathbf{e}_{f}, \quad \mathbf{c}_{2}=-\frac{f_{0} L^{2}}{12} \mathbf{e}_{f}
$$

In Table 2 the deflection vector $\mathbf{U}(L / 2)$ is listed for different values of $\alpha \quad\left(\mathbf{e}_{f}=\right.$ $\left.\cos \alpha \mathbf{e}_{x}+\sin \alpha \mathbf{e}_{y}\right)$. 
Table 2. Deflection vector of the midpoint of a fixed-fixed thin-walled non-homogeneous beam with open profile

\begin{tabular}{cccc}
\hline$\alpha$ & $\mathrm{U}(L / 2) / f_{0}\left[\frac{\mathrm{m}^{2}}{\mathrm{~N}}\right]$ & $\mathbf{U}_{B}(L / 2) / f_{0}\left[\frac{\mathrm{m}^{2}}{\mathrm{~N}}\right]$ & $\mathbf{U}_{S}(L / 2) / f_{0}\left[\frac{\mathrm{m}^{2}}{\mathrm{~N}}\right]$ \\
\hline 0 & $\left(\begin{array}{l}-5.64617 \mathrm{E}-06 \\
-2.44759 \mathrm{E}-06\end{array}\right)$ & $\left(\begin{array}{l}-5.7208 \mathrm{E}-06 \\
-2.4517 \mathrm{E}-06\end{array}\right)$ & $\left(\begin{array}{l}7.46312 \mathrm{E}-08 \\
4.10724 \mathrm{E}-09\end{array}\right)$ \\
\hline$\frac{\pi}{6}$ & $\left(\begin{array}{l}-6.11352 \mathrm{E}-06 \\
-3.87665 \mathrm{E}-06\end{array}\right)$ & $\left(\begin{array}{l}-6.18021 \mathrm{E}-06 \\
-3.89416 \mathrm{E}-06\end{array}\right)$ & $\left(\begin{array}{l}6.66862 \mathrm{E}-08 \\
1.75066 \mathrm{E}-08\end{array}\right)$ \\
\hline$\frac{\pi}{3}$ & $\left(\begin{array}{l}-4.94276 \mathrm{E}-06 \\
-4.26697 \mathrm{E}-06\end{array}\right)$ & $\left(\begin{array}{l}-4.98363 \mathrm{E}-06 \\
-4.29318 \mathrm{E}-06\end{array}\right)$ & $\left(\begin{array}{l}4.08726 \mathrm{E}-08 \\
2.62151 \mathrm{E}-08\end{array}\right)$ \\
\hline$\frac{\pi}{2}$ & $\left(\begin{array}{l}-2.44759 \mathrm{E}-06 \\
-3.51395 \mathrm{E}-06\end{array}\right)$ & $\left(\begin{array}{l}-2.4517 \mathrm{E}-06 \\
-3.54185 \mathrm{E}-06\end{array}\right)$ & $\left(\begin{array}{l}4.10724 \mathrm{E}-09 \\
2.78993 \mathrm{E}-08\end{array}\right)$ \\
\hline$\frac{5 \pi}{6}$ & $\left(\begin{array}{l}3.66593 \mathrm{E}-06 \\
3.62699 \mathrm{E}-07\end{array}\right)$ & $\left(\begin{array}{l}3.72851 \mathrm{E}-06 \\
3.52307 \mathrm{E}-07\end{array}\right)$ & $\left(\begin{array}{c}-6.25789 \mathrm{E}-08 \\
1.03927 \mathrm{E}-08\end{array}\right)$ \\
\hline$\frac{2 \pi}{3}$ & $\left(\begin{array}{l}7.03408 \mathrm{E}-07 \\
-1.81938 \mathrm{E}-06\end{array}\right)$ & $\left(\begin{array}{c}7.37167 \mathrm{E}-07 \\
-1.84149 \mathrm{E}-06\end{array}\right)$ & $\left(\begin{array}{c}-3.37586 \mathrm{E}-08 \\
2.21079 \mathrm{E}-08\end{array}\right)$ \\
\hline
\end{tabular}

\section{Conclusions}

It has been shown that the governing formulas of bending and shear can be written in a coordinate-free invariant form for arbitrary non-homogeneous cross sections. New coordinate-free expressions are derived for the location of the centre of shear of thinwalled beams with open and closed profiles. Explicit coordinate-free formulas of shear rigidity tensors have been presented for thin-walled cross sections with open and closed profiles. Numerical examples illustrate that the principal directions of bending and shear deformations may not be the same. A vector-tensor formulation of the first-order shear deformation theory for non-homogeneous thin-walled beams is also presented.

Acknowledgements. This research was (partially) carried out in the framework of the Center of Excellence of Innovative Engineering Design and Technologies at the University of Miskolc.

\section{REFERENCES}

1. WANG, C.-C.: Some vector formulas for the bending and shearing of prismatic beams with arbitrary cross section. Thin-Walled Structures, 21, (1995), 175-190.

2. Stokes, V. K.: A note on the pure bending of unsymmetrical prismatic beams. ASME Journ. of Appl. Mech., 44, (1977), 344-345.

3. Stokes, V. K.: Design with non-homogeneous materials - part i: Pure bending of prismatic bars. Trans. ASME Journ. of Vibration, Stress $\mathcal{E}$ Reliability in Design, 44, (1987), 82-86.

4. Stokes, V. K.: Thermoelastic pure bending of non-homogeneous prismatic bars. Journal of Thermal Stresses, 14, (1991), 499-518. 
5. Romano, G., Rosati, L., and Ferro, G.: Shear deformability of thin-walled beams of arbitrary cross sections. International Journal for Numerical Methods in Engineering, 35, (1992), 283-306.

6. LurJe, I.: Theory of Elasticity. Fiz-Mat. Lit, Moscow, 1970. In Russian.

7. Malvern, L. E.: Introduction to the Mechanics of a Continuous Medium. Prentice Hall, New York, 1969.

8. Mase, G. T. and Mase, G. E.: Continuum Mechanics. CRC Press, London, 1999.

9. Beer, F. P. and Johnston, E. R.: Mechanics of Materials. McGraw-Hill, New York, 1987.

10. Vlasov, V. Z.: Thin-Walled Elastic Beams. Israel Program for Scientific Translations. Jerusalem, Israel, 2nd edn., 1961.

11. Fung, Y. C.: Introduction to the Theory of Aeroelasticity. John Wiley, New York, 1955.

12. Wang, C. M., Reddy, J. N., and Lee, K. H.: Shear Deformable Beams and Plates. Relationships with Classical Solutions. Elsevier, New York, 2000. 